\title{
Heterogeneity of proangiogenic features in mesenchymal stem cells derived from bone marrow, adipose tissue, umbilical cord, and placenta
}

Wen Jing Du ${ }^{1 \dagger}$, Ying Chi ${ }^{1 \dagger}$, Zhou Xin Yang ${ }^{1}$, Zong Jin Li ${ }^{2}$, Jun Jie Cui ${ }^{1}$, Bao Quan Song ${ }^{1}$, Xue $\mathrm{Li}^{1}$, Shao Guang Yang ${ }^{1}$, Zhi Bo Han ${ }^{1 *}$ and Zhong Chao Han ${ }^{1,2^{*}}$

\begin{abstract}
Background: Mesenchymal stem cells (MSCs) have been widely proven effective for therapeutic angiogenesis in ischemia animal models as well as clinical vascular diseases. Because of the invasive method, limited resources, and aging problems of adult tissue-derived MSCs, more perinatal tissue-derived MSCs have been isolated and studied as promising substitutable MSCs for cell transplantation. However, fewer studies have comparatively studied the angiogenic efficacy of MSCs derived from different tissues sources. Here, we evaluated whether the in-situ environment would affect the angiogenic potential of MSCS.
\end{abstract}

Methods: We harvested MSCs from adult bone marrow (BMSCs), adipose tissue (AMSCs), perinatal umbilical cord (UMSCs), and placental chorionic villi (PMSCs), and studied their "MSC identity" by flow cytometry and in-vitro trilineage differentiation assay. Then we comparatively studied their endothelial differentiation capabilities and paracrine actions side by side in vitro.

Results: Our data showed that UMSCs and PMSCs fitted well with the minimum standard of MSCs as well as BMSCs and AMSCs. Interestingly, we found that MSCs regardless of their tissue origins could develop similar endothelial-relevant functions in vitro, including producing eNOS and uptaking ac-LDL during endothelial differentiation in spite of their feeble expression of endothelial-related genes and proteins. Additionally, we surprisingly found that BMSCs and PMSCs could directly form tubular structures in vitro on Matrigel and their conditioned medium showed significant proangiogenic bioactivities on endothelial cells in vitro compared with those of AMSCs and UMSCs. Besides, several angiogenic genes were upregulated in BMSCs and PMSCs in comparison with AMSCS and UMSCs. Moreover, enzyme-linked immunosorbent assay further confirmed that BMSCs secreted much more VEGF, and PMSCs secreted much more HGF and PGE2.

Conclusions: Our study demonstrated the heterogeneous proangiogenic properties of MSCs derived from different tissue origins, and the in vivo isolated environment might contribute to these differences. Our study suggested that MSCs derived from bone marrow and placental chorionic villi might be preferred in clinical application for therapeutic angiogenesis.

Keywords: Mesenchymal stem cells, Bone marrow, Adipose tissue, Umbilical cord, Placental chorionic villi, Pro-angiogenic features, Heterogeneity

\footnotetext{
*Correspondence: zhibohan@163.com; hanzhongchao@hotmail.com

${ }^{\dagger}$ Equal contributors

${ }^{1}$ The State Key Laboratory of Experimental Hematology, Institute of

Hematology and Hospital of Blood Disease, Chinese Academy of Medical

Science \& Peking Union Medical College, No. 288, Nanjing Road, Heping

District, Tianjin 300020, China

Full list of author information is available at the end of the article
} 


\section{Background}

Mesenchymal stem cells (MSCs) are able to self-renew and differentiate into a lineage of mesenchymal cells, such as adipocytes, osteocytes, and chondrocytes. Besides, they are of low immunogenicity and can exert potent immunosuppression on HLA-mismatched PBMCs, which make them ideal candidates for cell therapy and tissue engineering [1]. Moreover, MSCs have been widely reported to exert angiogenesis in in-vitro and in-vivo experiments [2]. Also, human bone marrow-derived MSCs (BMSCs) have been proven effective in clinical application for therapeutic angiogenesis in patients with critical limb ischemia [3]. Because of their favorite angiogenic properties, MSCs are attractive in clinical cell therapy and tissue engineering for ischemia disease treatment [4].

MSCs were first isolated from bone marrow in 1976 [5], and other tissue-derived MSCs were then later harvested in succession. During the last decades, MSCs derived from perinatal tissues [6], including umbilical cord blood, umbilical cord, and placenta, have attracted attention because of their noninvasive isolation methods and minimal ethical issues. Moreover, perinatal tissuederived MSCs are young cells without higher possibilities of incorporated mutation in comparison with adult tissue-derived MSCs [6].

Many studies have comparatively analyzed the differential properties and biological functions of MSCs derived from perinatal and adult tissues, including their molecule profile [7], tridifferentiation potentials [8], proliferation/clonogenic formation capacities [9], immunomodulatory functions [10], and hematopoietic support abilities [11]. However, fewer studies have so far investigated their angiogenic differences. Previous studies have shown that both adult BMSCs and adipose tissue-derived MSCs (AMSCs) could induce remarkable therapeutic angiogenesis [12]. Umbilical cord-derived MSCs (UMSCs) and placental chorionic villi-derived MSCs (PMSCs) have also been reported to display angiogenic activity in invitro and in-vivo experiments [13]. However, under the same culture conditions, do these perinatal MSC populations have similar angiogenic effects as the typical adult BMSCs and AMSCs? Does the in-situ environment of MSCs affect their angiogenic properties? Is there any influence of the development stage on their angiogenic functions? To answer these questions, we designed this project and comparatively analyzed the angiogenic differences of BMSCs, AMSCs, UMSCs, and PMSCs.

\section{Methods}

\section{Cell culture}

The study was approved by the Ethical Committee and the Institutional Review Board of the Chinese Academy of Medical Science \& Peking Union Medical College. Bone marrow was collected from healthy volunteers in the Blood Diseases Hospital. BMSCs were isolated following the protocol described previously [14]. AMSCs, UMSCs, and PMSCs at passage 1 were supplied by the Cell Products of National Engineering Research Center (http://www.amcellgene.com) [15, 16]. Additionally, one pair of UMSCs and PMSCs that derived from the same donor was used. All volunteers provided informed consent. BMSCs, AMSCs, UMSCs, and PMSCs were cultured in the same standard culture condition. The standard complete culture medium for MSCs was DMEM/F12 (DF12; Gibco, Grand Island, NY, USA), 10 \% fetal bovine serum (FBS; HyClone), $2 \mathrm{mM}$ glutamine (Sigma, St. Louis, MO, USA), $100 \mathrm{U} / \mathrm{ml}$ penicillin-streptomycin (P/S; Invitrogen, Carlsbad, CA, USA), and $10 \mathrm{ng} / \mathrm{ml}$ epidermal growth factor (EGF; Peprotech). Human umbilical vein endothelial cells (HUVECs) were harvested by digesting umbilical cord vein for 15 minutes at $37^{\circ} \mathrm{C}$. HUVECs were cultured in Endothelial Growth Medium-2MV (EGM-2MV; Lonza, Walkersville, MD, USA), and HUVECs at passage 3 5 were used for experiments.

\section{Endothelial differentiation}

Cells were seeded on Matrigel (1:100 dilution; BD Bioscience, Bedford, MA, USA) precoated flasks, and cultured with EGM2-MV supplemented with $50 \mathrm{ng} / \mathrm{ml}$ VEGF (Prepotech) [17] for 2 weeks. Endothelial differentiation medium was changed twice a week. Cells cultured in MSC complete medium were used as the control. Cells with or without endothelial differentiation were then harvested, and the expression of endothelial-related genes was independently tested by RT-PCR and immunostaining.

\section{Real-time PCR}

Endothelial-related genes were respectively tested on undifferentiated and differentiated MSCs, and their relative expression level in undifferentiated MSCs was normalized to 1; thus the fold-change in gene expression of differentiated MSCs was calculated as $2^{-\Delta \Delta C T}$. Additionally, to comparatively analyze the expression level of angiogenic cytokines on BMSCs, AMSCs, UMSCs, and PMSCs, RT-PCR was also performed. The relative expression level of the candidate gene in particular BMSCs or PMSCs was normalized to 1 , and its relative expression fold in other cells was shown as $2^{-\Delta \Delta C T}$. The real-time PCR was performed as follows: total RNA was extracted using the E.Z.N.A. Total RNA Kit I (OMEGA, Norcross, GA, USA), and cDNA was then synthesized by using the MLV RT Kit (Invitrogen). The SYBR Green detection method was employed, and the Applied Bio system 7900 or 7300 Real-Time PCR System (Foster City, CA, USA) was used. Each sample was performed in triplicate $(n=3-5)$. Primers involved are listed in Additional file 1: Table S1. 


\section{Immunostaining}

EC-differentiated MSCs were harvested and seeded at $2 \times 10^{4}$ cells $/ \mathrm{cm}^{2}$ in glass-bottom cell culture dishes (NEST) overnight. Cells were first fixed with $4 \%$ paraformaldehyde (PFA) for 15 minutes. After permeabilization with $0.25 \%$ Triton X-100 solution (Sigma) for 30 minutes, the nonspecific epitope of cells was blocked by $0.2 \%$ albumin bovine $\mathrm{V}$ for 30 minutes. The cells were then incubated with mouse anti-human von Willebrand Factor (vWF, 1:100 dilution; Abcam, Cambridge, MA, USA) or rabbit anti-human eNOS antibodies (1:100 dilution; Abcam) for 60 minutes, followed by staining with FITCconjugated anti-rabbit or anti-mouse antibodies (1:100 dilution; Invitrogen, Molecular Probes) for 30 minutes. Cells stained with FITC-conjugated anti-mouse or antirabbit IgG alone served as the control. Finally, cells were stained with DAPI for 5 minutes, fixed with $2 \%$ PFA, and then photographs were taken by PerkinElmer UltraVIEW Vox confocal microscope (PerkinElmer, Waltham, MA, USA) under $\times 200$ magnification.

\section{Acetylated low-density lipoprotein-uptaking assay}

To assess whether cells after endothelial differentiation developed the endothelial-specific function in terms of uptaking acetylated low-density lipoprotein (ac-LDL), the acLDL-uptaking assay was performed. We harvested EC-differentiated MSCs and seeded them on small culture dishes with a glass bottom (NEST). After overnight incubation in serum-free culture medium, cells were cultured in fresh complete medium with $10 \mu \mathrm{g} / \mathrm{ml}$ Dil-acLDL (Invitrogen) for 4 hours. Later, cells were washed twice with PBS and fixed with $4 \%$ PFA following DAPI staining. HUVECs were used as positive control. Photographs were taken using a PerkinElmer UltraVIEW Vox confocal microscope.

\section{Conditioned medium preparation}

MSCs were plated at $4 \times 10^{4}$ cells $/ \mathrm{cm}^{2}$ in a T25 flask overnight. After washing twice with PBS, cells were cultured with $10 \mathrm{ml}$ EBM2 (Lonza) for another 2 days. Their conditioned media (CMs) were then collected, centrifuged at $400 \times g$ for 10 minutes to remove the cell debris, filtered through a $0.2 \mu \mathrm{m}$ filter (Pall Corporation, Ann Arbor, MI, USA), and frozen at $-80{ }^{\circ} \mathrm{C}$ for further studies. MSCs derived from three donors were used.

\section{In-vitro Matrigel tube formation assay} Direct Matrigel tube formation assay

To investigate their angio-vasculogenic capacities [18], BMSCs, AMSCs, UMSCs, and PMSCs were collected and seeded directly on a Matrigel (BD Bioscience) precoated 96-well plate at $2 \times 10^{4}$ cells/well in MSC complete medium. Photographs were taken using the microscope (Olympus, Melville, NY, USA) after 12 hours of incubation (scale bar $=500 \mu \mathrm{m})$. Tube numbers in each well were counted and each sample was performed in triplicate (BMSCs, $n=2$; AMSCs, UMSCs, and PMSCs, $n=3$ ).

\section{Indirect Matrigel tube formation assays}

To better study the paracrine action of BMSCs, AMSCs, UMSCs, and PMSCs, we used their CMs to incubate endothelial cells and further assessed their trophic effects on the angiogenic function of endothelial cells. CMs supplemented with $2 \%$ FBS, EBM2 supplemented with $2 \%$ FBS (served as the negative control), and EGM2-MV (contained plentiful cytokines and $2 \%$ FBS, served as the positive control) were used to culture endothelial cells for 9 hours, respectively. Endothelial cells were seeded on Matrigel at $2 \times 10^{4}$ cells/well in a 96-well plate. Photographs were taken by microscope (scale bar $=500 \mu \mathrm{m}$ ). Tube numbers in each well were counted, and the total tube length and total tube area in each well were measured using ImageJ software (NIH, USA). Three donorderived CMs were used and each sample was performed in duplicate.

\section{Endothelial cell proliferation assay}

To investigate the proproliferative effects of the secretion of BMSCs, AMSCs, UMSCs, and PMSCs, endothelial cells were incubated with different MSC population-derived CMs. The endothelial cell proliferation was measured using the Cell Counting Kit-8 (Dojindo, Rockville, MD, USA). Endothelial cells were first seeded at $1.0 \times 10^{4}$ cells/well in a 96-well plate overnight. After gently removing the medium, cells were washed twice with PBS, and then CMs supplemented with $2 \%$ FBS, EBM2 supplemented with $2 \%$ FBS (served as the negative control), and EGM2-MV (served as the positive control) were added to the cells for another 48 hours. Each sample was performed in triplicate. $\triangle \mathrm{OD} 450$ indicated the final data after subtracting the background.

\section{Enzyme-linked immunosorbent assay}

To determine the concentration of VEGF, HGF, bFGF, and PGE2 in supernatants of MSCs, enzyme-linked immunosorbent assays (ELISAs) were performed. The 48-hour supernatants of MSCs with an initial seeding density of 20,000 cells/well in a 96-well plate were collected for VEGF, HGF, and bFGF measurements, while the 72-hour supernatants of MSCs with an initial density of $10^{5}$ cells/well in a six-well plate were collected for PGE2 measurements. All of the supernatants were centrifuged at $400 \times g$ for 10 minutes and then measured by their corresponding ELISA kits. The ELISA kits for VEGF, HGF, and bFGF were purchased from Neobioscience Biotech (Shenzhen, China), and the PGE2 ELISA kit was purchased from Cayman Chemicals. All of the procedures 
strictly followed the corresponding instructions. Supernatants derived from three donors were used.

\section{Statistical analysis}

Statistical analysis was performed by GraphPad Prism 6.0 software (Graph Pad Software, Inc., San Diego, CA, USA). All data are shown as the mean \pm SEM. One-way ANOVA followed by Bonferroni multiple comparisons was employed to determine the statistical significance. Paired $t$ test was used to analyze the endothelial gene modification after endothelial differentiation. The result was considered statistically significant if $p<0.05$.

\section{Results}

\section{Limited potentials of endothelial differentiation}

Previous studies have shown that the endothelial differentiation mechanism participates in MSC-mediated wound healing [19]. To investigate their capabilities of endothelial differentiation, BMSCs, AMSCs, UMSCs, and PMSCs were harvested and induced into endothelial cells in vitro for 2 weeks. The undifferentiated and differentiated MSCs were then collected, and the endothelial gene expression further comparatively analyzed. Data revealed that the relative expression levels of CD31, CD34, Flt1, vWF, $V E$-Cadherin $(V E C)$, and Tie-2 were altered differently in EC-differentiated MSCs in comparison with undifferentiated cells; however, no statistical significance was found $(p>0.05)$ (Fig. 1a). There was an increase in expression of $C D 31, C D 34, v W F$, and VEC in EC-differentiated AMSCs, UMSCs, and PMSCs but a decreased expression in EC-differentiated BMSCs. Similarly, Flt-1 was upregulated in AMSCs and UMSCs but declined in BMSCs and PMSCs after endothelial differentiation. Tie-2 expression was raised to various degrees in BMSCs, AMSCs, and PMSCs during endothelial differentiation, but with a falloff in UMSCs. To better define the expression of endothelial-related proteins and the unique functions of cells after endothelial differentiation, an immunostaining assay $[20,21]$ and an acLDL-uptaking assay [22] were performed respectively (Fig. 1b). Our data showed that EC-differentiated MSCs weakly expressed vWF and CD31 in contrast to the HUVECs (positive control). However, MSCs produced eNOS and developed acLDL uptaking capacities to some extent after endothelial differentiation, which were special functions of endothelial cells. This observation indicated that MSCs could develop some properties of endothelial cells under appropriate conditions.

Heterogeneous angio-vasculogenic capacities of BMSCs, AMSCs, UMSCs, and PMSCs on in-vitro Matrigel tube formation assay

The in-vitro Matrigel tube formation assay was generally used as the first screen to test whether a compound might participate in angiogenesis [23]. MSCs were reported to spontaneously generate 3-D capillary-like structures on Matrigel in vitro $[18,24]$. To determine their angio-vasculogenic capacities, BMSCs, AMSCs, UMSCs, and PMSCs were directly seeded on Matrigel and the tube formation was observed after 12 hours of incubation. Interestingly, intact tube structures were seen in the BMSC and PMSC groups rather than in the groups of AMSCs and UMSCs (Fig. 2a). Tube numbers in the BMSC and PMSC groups were $11.65 \pm 2.92$ and $6.49 \pm$ 1.18 , respectively, much higher than those in the AMSC and UMSC groups $(0.91 \pm 0.76$ and $0.41 \pm 0.20, p<0.05)$ (Fig. 2b). These observations indicated that BMSCs and PMSCs had better angio-vasculogenic capacities in comparison with UMSCs and AMSCs.

\section{BMSCs and PMSCs displayed potent paracrine actions on endothelial cells}

Secreted factors from MSC populations have been reported to significantly enhance the proliferation and function of endothelial cells in vitro [25]. To comparatively analyze their paracrine actions, we respectively used $\mathrm{BMSC}^{\mathrm{CM}}$, $\mathrm{AMSC}^{\mathrm{CM}}$ UMSC $^{\mathrm{CM}}$, and $\mathrm{PMSC}^{\mathrm{CM}}$ to culture endothelial cells and comparatively analyzed the effects on the proliferation and tube formation capacity of endothelial cells. We found that BMSC ${ }^{\mathrm{CM}}$ and PMSC ${ }^{\mathrm{CM}}$ could significantly promote endothelial cell proliferation $(1.23 \pm 0.06$ and $1.24 \pm 0.06)$ in contrast to $\mathrm{AMSC}^{\mathrm{CM}}$ and $\mathrm{UMSC}^{\mathrm{CM}}(0.84 \pm 0.02$ and $0.85 \pm 0.07 ; n=3, * p<0.05$ and $\left.{ }^{* *} p<0.01\right)$ during 48 hours of incubation. The proproliferative effects of $\mathrm{BMSC}^{\mathrm{CM}}$ and $\mathrm{PMSC}^{\mathrm{CM}}$ were similar to EGM2-MV (the complete endothelial cell culture medium containing plentiful growth factors), and much greater than EBM2 $(0.74 \pm 0.03, p<0.01)$. However, AMSC ${ }^{\mathrm{CM}}$ and $\mathrm{UMSC}^{\mathrm{CM}}$ did not reveal such promotion on the mitosis of endothelial cells compared with the EBM2 group $(n=3, p>0.05)$. Hence, we supposed that BMSCs and PMSCs secreted many more proproliferative factors than AMSCs and UMSCs (Fig. 3a). In addition, we used $\mathrm{BMSC}^{\mathrm{CM}}$, $\mathrm{AMSC}^{\mathrm{CM}}$, $\mathrm{UMSC}^{\mathrm{CM}}$, and $\mathrm{PMSC}^{\mathrm{CM}}$ to culture endothelial cells to test whether the secreted factors would affect the tube formation capacities of endothelial cells on Matrigel in vitro. Interestingly, CMs significantly promoted tube formation of endothelial cells by contrast with EBM2, in which no intact tube structure was observed (Fig. 3b). Similarly, we found $\mathrm{BMSC}^{\mathrm{CM}}$ and $\mathrm{PMSC}^{\mathrm{CM}}$ significantly promoted endothelial cells to generate intact tube structures, which numbered $30.33 \pm 3.59$ and $31.17 \pm 2.18$, respectively, much greater than those in the group of $\mathrm{AMSC}^{\mathrm{CM}}$ and $\mathrm{UMSC}^{\mathrm{CM}}(17.50 \pm 2.09$ and $18.50 \pm 1.38 ; n=3, " p<0.05$ and ${ }^{* *} p<0.01$ ) (Fig. 3c). The total tube area and total tube length were also greater in the $\mathrm{BMSC}^{\mathrm{CM}}$ and $\mathrm{PMSC} \mathrm{CM}^{\mathrm{CM}}$ groups (BMSC ${ }^{\mathrm{CM}}$ : $11.90 \pm 1.46$ and $75.93 \pm 8.83$; $\mathrm{PMSC}^{\mathrm{CM}}$ : 


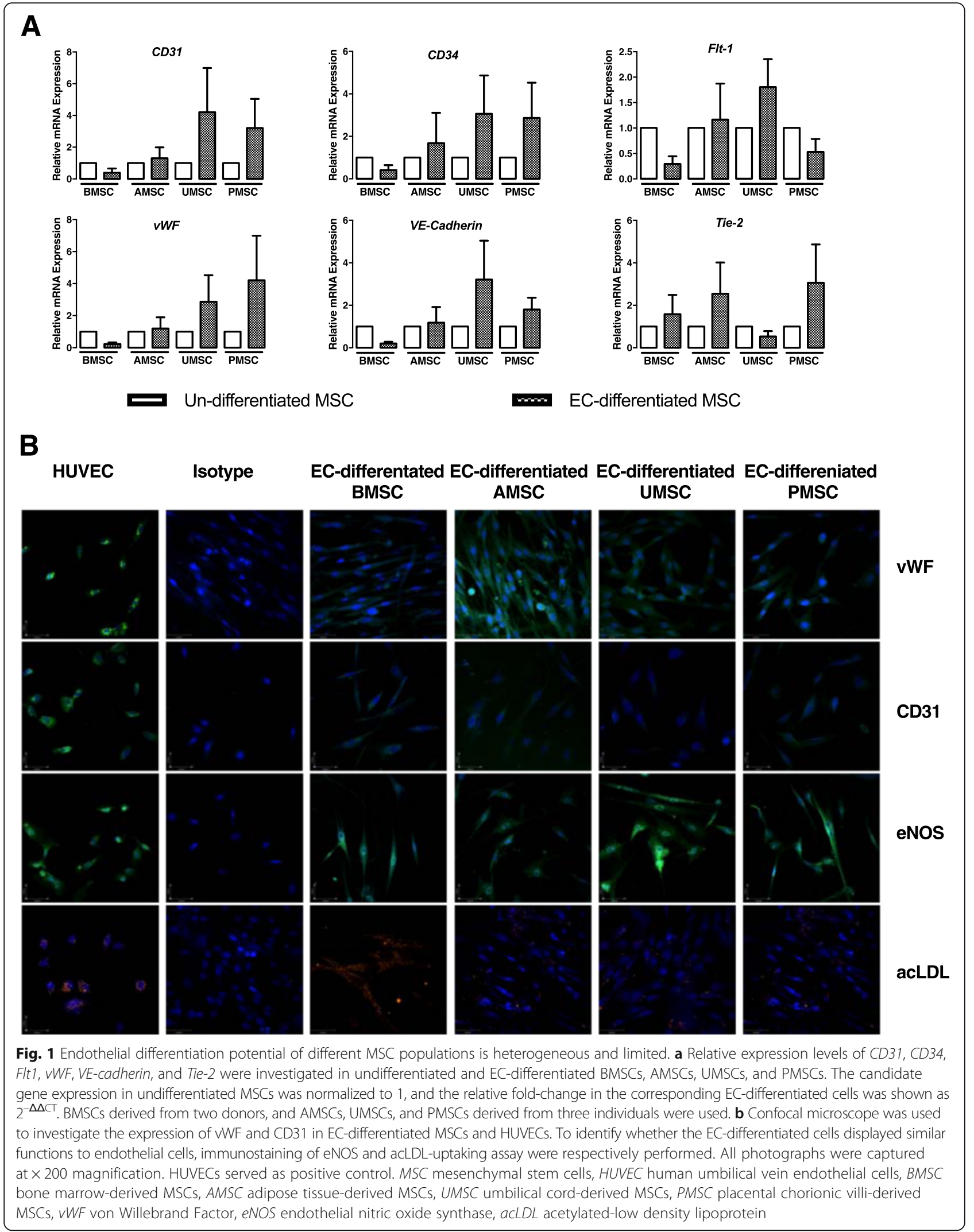



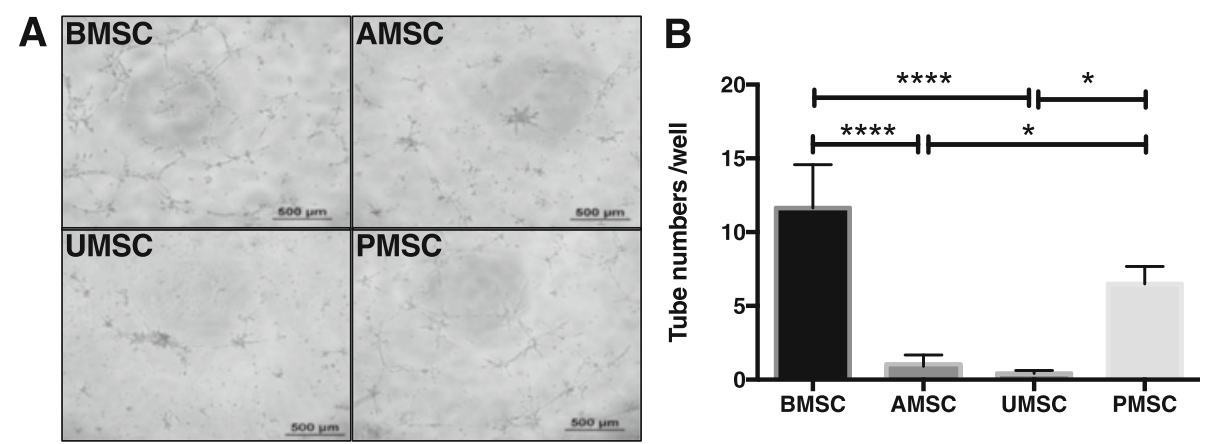

Fig. 2 MSCs derived from different tissue sources display distinct tube formation capacities on Matrigel in vitro. MSCs were seeded on Matrigel in vitro at $2 \times 10^{4}$ cells/well. MSCs could form tube-like structures on Matrigel temporarily (a). Representative photographs were taken after 12 hours of incubation (scale bar $=500 \mu \mathrm{m}$ ). b Numbers of tube structures were counted and analyzed (BMSC, $n=2$; AMSC, UMSC, and PMSC, $n=3)$. Each sample was performed in triplicate $\left({ }^{*} p<0.05,{ }^{* * *} p<0.0001\right)$. MSC mesenchymal stem cells, BMSC bone marrow-derived MSCs, AMSC adipose tissue-derived MSCs, UMSC umbilical cord-derived MSCs, PMSC placental chorionic villi-derived MSCs

$13.39 \pm 0.70$ and $83.36 \pm 4.73)$ than in the $\mathrm{AMSC}^{\mathrm{CM}}$ and UMSC $^{\mathrm{CM}}$ groups (AMSC ${ }^{\mathrm{CM}}$ : $7.20 \pm 1.12$ and $45.05 \pm 5.39$; UMSC $^{\mathrm{CM}}: 9.03 \pm 0.39$ and $51.16 \pm 2.36 ; n=3$, $* 0<0.05$, ** $p<0.01$ and *** $p<0.001$ ) (Fig. 3d, e). These results indicated that the secretion of BMSCs and PMSCs contained more bioactive factors than that of AMSCs and UMSCs in the enhancement of the vasculogenic functions of endothelial cells.

\section{PMSCs and BMSCs expressed higher levels of angiogenic factors}

Cytokines secreted by MSCs played vital roles in the process of MSC-mediated angiogenesis [26]. Referring to the previous research $[25,26]$, we selected a panel of candidates, including VEGF-A, VEGF-C, HGF, bFGF, NGF, angiogenin (ANG), TGF- $\beta, I L-6$ [27], IL-8 [28], IL-1 $\alpha$ [29], $I L-1 \beta$ [30], and Cox-2 [31], and tested their relative expression levels in different MSC populations. Oneway ANOVA analysis revealed that the comparison of VEGF- $A, H G F, b F G F, N G F, I L-6, I L-8, T G F-\beta, I L-1 \alpha, I L-$ $1 \beta$, and Cox-2 expression among different MSC types was statistically significant $(p<0.05)$; while the expression of VEGF-C, ANG, and TGF- $\beta$ in different MSC populations showed no significant difference $(p>0.05)$. Of note, the relative expression levels of $H G F, b F G F, I L-6$, $I L-8, I L-1 \alpha, I L-1 \beta$, and Cox-2 in PMSCs were comparatively higher than that in other MSC populations. Besides, BMSCs expressed a comparatively higher level of $V E G F-A, N G F$, and ANG (Fig. 4a). Therefore, PMSCs, as well as BMSCs, were superior to AMSCs and UMSCs for angiogenic gene expression.

As previous studies have reported, bFGF, VEGF, HGF, and PGE2 could significantly promote angiogenesis $[32,33]$. To further explore the secretion of angiogenicrelated factors, we assessed the concentrations of bFGF, VEGF, HGF, and PGE2 in supernatants of different MSC populations via ELISA. Data showed that MSCs secreted a lower level of bFGF under normal conditions, which was about 36-69 pg/ml. The concentrations of VEGF in the supernatants of BMSCs and PMSCs were $882.7 \pm$ $55.27 \mathrm{pg} / \mathrm{ml}$ and $721.4 \pm 120.4 \mathrm{pg} / \mathrm{ml}$, respectively, much higher than that in UMSC-derived supernatants (77.16 \pm $77.16 \mathrm{pg} / \mathrm{ml} ; n=3, p<0.01)$, and a medial level of VEGF $(486.3 \pm 111.8 \mathrm{pg} / \mathrm{ml})$ was found in AMSC supernatant. Furthermore, the HGF concentration in supernatants of perinatal tissue-derived MSCs (PMSCs: 9466 pg/ml; UMSCs: $7694 \mathrm{pg} / \mathrm{ml}$ ) was significantly higher than that in adult tissue-derived MSCs (BMSCs: $119 \mathrm{pg} / \mathrm{ml}$; AMSCs: $1589 \mathrm{pg} / \mathrm{ml})(n=3, p<0.05$; Fig. 4b), which was in accordance with our RT-PCR results and also agreed with the study by Amable et al. [34]. Additionally, the PGE2 concentration in supernatants of PMSC $(32,628 \mathrm{pg} / \mathrm{ml})$ was significantly higher than that in other MSC types (BMSC: $2074 \mathrm{pg} / \mathrm{ml}$, AMSC: $337.9 \mathrm{pg} / \mathrm{ml}$; UMSC: $3603 \mathrm{pg} / \mathrm{ml} ; n=3, p<0.05)$.

To further study whether the in-situ environment would affect the angiogenic properties of MSCs, we evaluated the relative expression of $I L-8, H G F, T G F-\beta, I L-1 \alpha$, $I L-1 \beta, I L-6$, and Cox-2 in one donor-derived UMSC and PMSC by RT-PCR. Our results indicated that PMSCs expressed a remarkably higher level of $I L-8, H G F, I L-1 \alpha$, $I L-1 \beta, I L-6$, and Cox-2 than UMSCs (Fig. 4c). Thus, considering the possible impact of their different isolated environment, placental chorionic villi would be a favorite tissue source for isolating MSCs with superior angiogenic cytokine expression.

\section{Discussion}

In the present study, we performed a parallel comparison of angiogenic potentials of MSCs derived from adult tissues (bone marrow and adipose tissue) and perinatal tissues (umbilical cord and placental chorionic villi). Cells were isolated from different in-situ environments as well as distinct developmental stages, which we hypothesized 


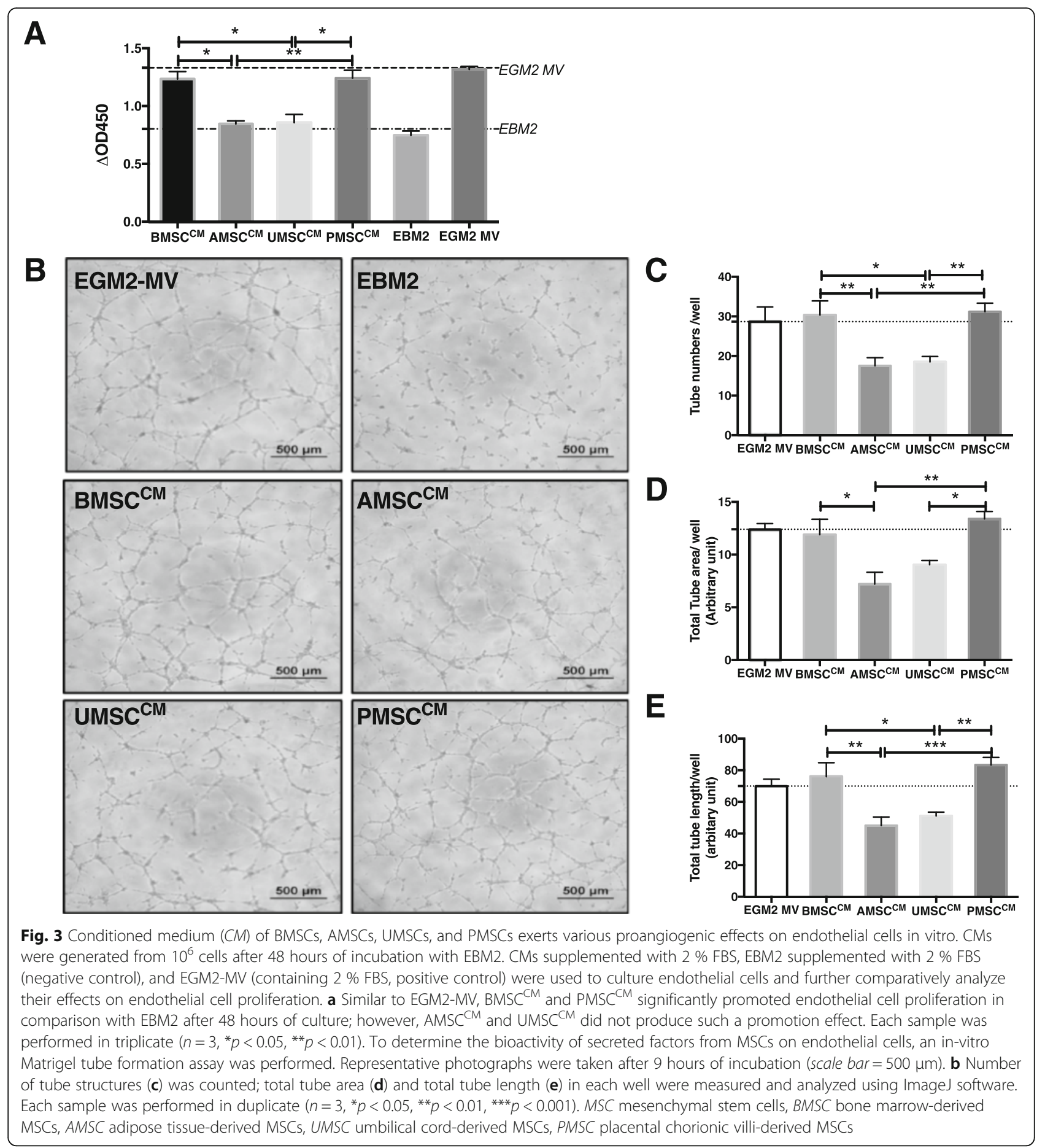

might affect their angiogenic properties. After verifying the phenotype and trilineage differentiation, we comparatively studied their angiogenic properties from two aspects: endothelial differentiation and paracrine activity. MSCs regardless of their tissue origins have limited capacities to transform into endothelial cells; however, the $\mathrm{CM}$ of BMSCs and PMSCs showed stronger proangiogenic activities on endothelial cells in vitro than that of AMSCs and UMSCs.

The endothelial differentiation capacity of MSCs remains controversial. Aguilera et al. [20] demonstrated that Wharton's jelly-derived mesenchymal cells expressed high levels of endothelial markers (CD31 and KDR) and also synthesized NO after 30 days of endothelial 


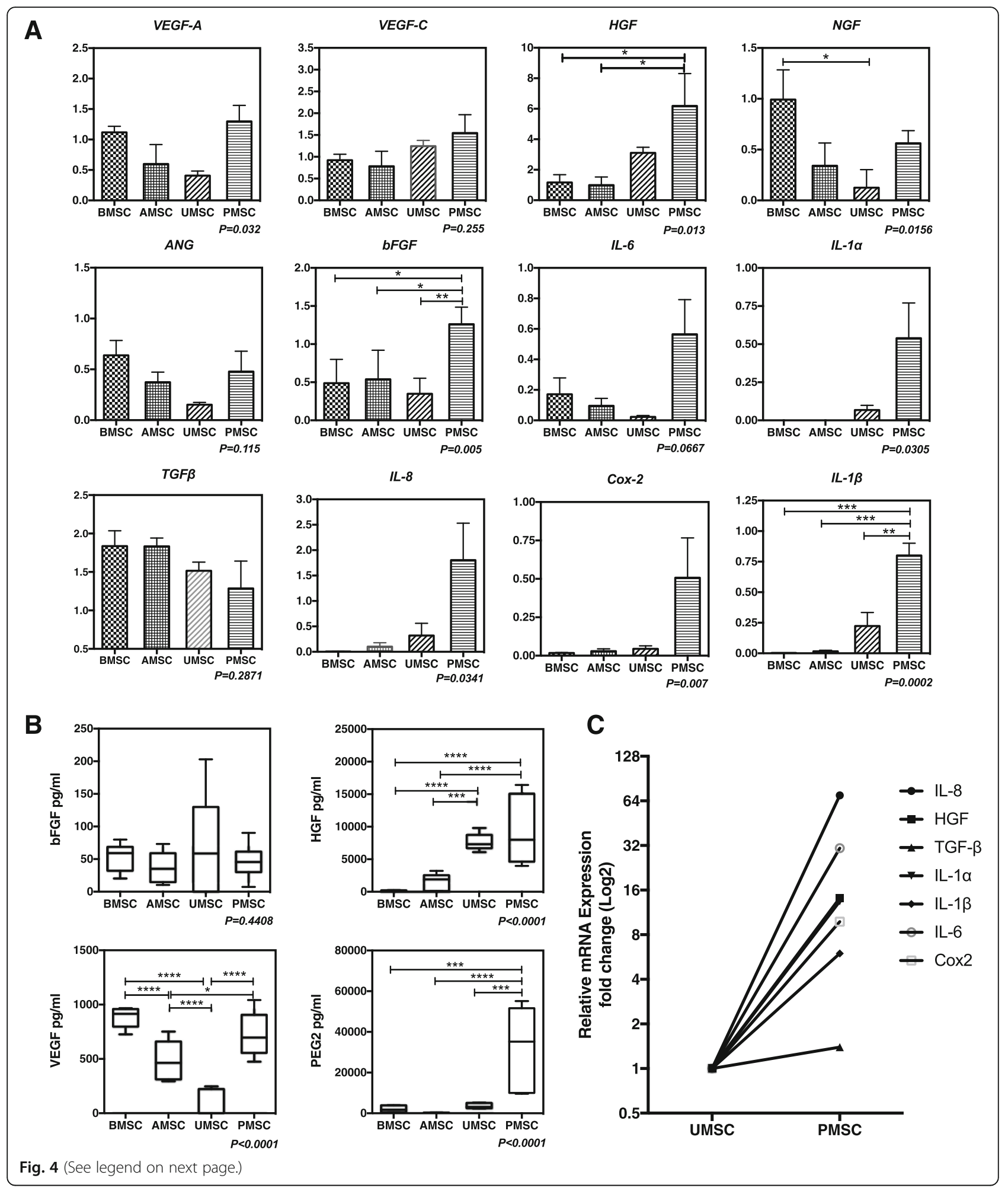




\section{(See figure on previous page.)}

Fig. 4 Gene and protein expression of selected paracrine factors in different MSC populations. a Relative mRNA expression of selected angiogenic cytokines was assessed, including VEGF-A, VEGF-C, HGF, bFGF, NGF, ANG, TGF- $\beta, I L-6, I L-8, I L-1 a, I L-1 \beta$, and Cox-2. The relative expression level of the candidate cytokine in one sample of BMSCs or PMSCs was normalized to 1, and all other samples for corresponding gene expression were shown as $2^{-\Delta \Delta C T}$ fold-change. Each sample was tested in triplicate $(n=3-5)$. b Concentrations of bFGF, VEGF, and HGF in 48-hour supernatants and the PGE2 concentration in 72-hour supernatants of BMSCs, AMSCs, UMSCs, and PMSCs were measured using corresponding ELISA kits $\left(n=3,{ }^{*} p<0.05\right.$, $\left.{ }^{* *} p<0.01\right)$. c The relative mRNA expression of $I L-8, H G F, T G F-\beta, I L-1 a, I L-1 \beta, I L-6$, and Cox-2 was separately tested on one donor-derived UMSC and PMSC. The relative expression level of the candidate gene in UMSCS was normalized to 1 , and the fold-change in PMSCS was expressed as $2^{-\triangle \Delta C T}$. To better show the different expression of angiogenic genes in UMSCs and PMSCs, data were shown in $\log _{2}$. MSC mesenchymal stem cells, BMSC bone marrow-derived MSCS, AMSC adipose tissue-derived MSCs, UMSC umbilical cord-derived MSCs, PMSC placental chorionic villi-derived MSCs, VEGF vascular endothelial growth factor, HGF hepatocyte growth factor, bFGF basic fibroblast growth factor, NGF nerve growth factor, ANG angiogenin, TGF- $\beta$ transforming growth factor beta, IL interleukin

differentiation. Additionally, Ikhapoh et al. [35] and Benavides et al. [21] respectively reported that BMSC and amniotic fluid-derived stem cells were capable of differentiation into endothelial cells. By contrast, Choi et al. [36] reported that Wharton's jelly-derived mesenchymal stromal/stem cells did not express endothelial specific markers (VEGFR2, Tie2, vWF, CD31, and VEcadherin) when they were cultured in endothelial induction medium. Partially agreeing with their results, our data showed that EC-differentiated MSCs were not sufficient to transform into mature endothelial cells because of their very low expression levels of CD31. However, endothelial differentiation did prime MSCs by upregulating some endothelial-related genes and developing endothelial-special functions, such as eNOS generation and acLDL-uptaking capacity, which was in accordance with the study by Janeczek Portalska et al. [37]. Although all of the cells were cultured with endothelial differentiation medium under the same condition, an inconsistent change of endothelial-related genes was seen in our results, which might be due to the wide interdonor variability. A similar result was also seen in the study of Kim et al. [18]. Because the shear stress [38] as well as the small molecule or microgravity [39] could enhance the efficiency of MSC endothelial differentiation, a modified protocol might help MSCs transform into endothelial cells in vitro.

In-vivo MSC transplantation studies demonstrated that very few engrafted MSCs remained several weeks post transplantation. Wu et al. [19] reported that $27 \%$ of transplanted MSCs engrafted into the wounded skin but only $2.5 \%$ cells remained 28 days post administration. Moon et al. [40] demonstrated that less than $1 \%$ engrafted AMSCs were incorporated into the host vascular structures 4 weeks after cell transplantation. In view of the clinical application of MSCs, endothelial differentiation may not be the principal mechanism of MSC-mediated regeneration. Meanwhile, accumulating evidence suggested that the bioactive secretion of MSCs was the dominant reason for their therapeutic benefits [26]. Kwon et al. [33] demonstrated that the CM of human MSCs significantly improved the in-vitro angiogenic activity of endothelial cells and promoted the blood restoration in mice with hind-limb ischemia. Consistently, our result also confirmed the strong proangiogenic action of MSCs via in-vitro paracrine assays.

In addition, many studies have indicated heterogeneous angiogenic gene expression in different MSC types. For example, Hsiao et al. [25] demonstrated a similar mRNA expression level of VEGF-A, $b F G F, H G F$, and NGF in BMSCs and AMSCs. Amable et al. [34] reported that differential secretion was found in BMSCs, AMSCs, and Wharton's Jelly-derived MSCs, which was consistent with our result, especially on VEGF and HGF secretion. Similarly, Rehman et al. [41], Kandel and Pittenger [42], and Bronckaers et al. [26] also demonstrated a varying paracrine action in different tissue-derived MSCs. Here, our study determined a distinct angiogenic cytokine expression by different MSC types, particularly the secretion of VEGF, HGF, and PGE2. Previous studies have shown that VEGF [43], HGF [44], and PGE2 [32] were important angiogenic cytokines that could significantly promote the growth and tube formation of endothelial cells. Because the different paracrine actions may lead to distinct therapeutic outcomes, some tissue-derived MSC populations with potent paracrine activity should be preconsidering cell banking and clinical application. Our study showed heterogeneous paracrine behaviors of different MSC types, which may indicate potential for tissue source selection of MSCs for clinical therapeutic angiogenesis. Nevertheless, the in-vivo pro-angiogenic properties of different MSC types still need further exploration.

Our FACS results showed distinct vascular cell adhesion molecular-1 (VCAM-1) expression on different MSC types. VCAM-1, also known as CD106, is extensively expressed on endothelial cells [45] and some stromal cells in a particular niche, such as the vascular niche [46] and the hematopoietic niche [47, 48]. Wang et al. [49] described that MSCs pretreated with cytokines (IL-1 $\beta$ and TNF- $\alpha$ ) upregulated VCAM-1 expression and revealed an improved treatment effect on cardiovascular ischemia. Recently, our group reported that VCAM-1+ placental chorionic villi-derived MSCs secreted abundant angiogenic cytokines and displayed 
therapeutic angiogenesis on hind-limb ischemia nude mice [50]. These studies indicated a possible correlation between a higher VCAM-1 expression ratio on MSCs and a better proangiogenic activity. Consistently, our present study suggested that a better paracrine action existed in MSC types with a higher VCAM-1 expression level, such as PMSCs and BMSCs.

In addition, BMSCs, AMSCs, UMSCs, and PMSCs are respectively isolated from different in-vivo environments: bone marrow and placental chorionic villi are abundant with capillaries, adipose tissue is filled with adipocytes, and umbilical cord contains not only blood vessels but also Wharton's Jelly (the tissue source of UMSCs). These distinct in-vivo environments may affect the bioactivities of MSCs. Konig et al. [51] demonstrated that placental MSCs isolated from blood vessels were better than those from avascular tissues on supporting endothelial cell functions. Jeon et al. [52] reported that placenta-derived MSCs retained a higher therapeutic efficacy than BMSCs and AMSCs in the hind-limb ischemic disease model. Moreover, our RT-PCR results showed a higher angiogenic cytokine expression in the same donor-derived PMSCs rather than UMSCs, which further indicated that the in-situ environment but not the development status might have some effect on the proangiogenic features of MSCs. On the other hand, the in-vitro angiogenic features of MSCs may reflect the characteristics of their different in-situ environment to some extent.

\section{Conclusions}

In this study, we described the heterogeneous proangiogenic features of MSCs derived from different tissue sources, and further demonstrated that the in-vivo environment but not the development status might contribute to their functional heterogeneity. Moreover, our study suggested BMSCs and PMSCs might be preferred in clinical application for vascular diseases due to their potent paracrine actions.

\section{Additional file}

Additional file 1: Table S1. Primers for real-time PCR were listed as follows. (DOCX $1460 \mathrm{~kb}$ )

\footnotetext{
Abbreviations

acLDL: Acetylated-low density lipoprotein; AMSC: Adipose tissue-derived MSC; ANG: Angiogenin; ANOVA: Analysis of variance; bFGF: Basic fibroblast growth factor; BMSC: Bone marrow-derived MSC; CM: Conditioned medium; Cox-2: Cyclooxygenase-2; EGF: Epidermal growth factor; eNOS: Endothelial nitric oxide synthase; FBS: Fetal bovine serum; HGF: Hepatocyte growth factor; HLA: Human leukocyte antigen; HUVEC: Human umbilical vein endothelial cell; IBMX: 3-Isobutyl-1-methylxanthine; NGF: Nerve growth factor; P/S: Penicillin/streptomycin; PBMC: Peripheral blood mononuclear cell; PFA: Paraformaldehyde; PGE2: Proinflammatory prostaglandin E2; PMSCs: Placental chorionic villi-derived MSCs; TGF- 3 : Transforming growth factor beta; UMSC: Umbilical cord-derived MSC; VE-Cadherin: Vascular
}

endothelial cadherin; VEGF: Vascular endothelial growth factor; VWF: von Willebrand Factor

\section{Funding}

This study was supported by the Natural Science Foundation of China (81330015 and 31470951) and the National Science and Technology Support Program (2013BAI01B09).

\section{Availability of data and materials}

The datasets supporting the conclusions of this article are included within the article.

\section{Authors' contributions}

WJD and YC carried out the conception, designed the experiment, and drafted the manuscript. WJD, JJC, and ZXY performed the FACS analysis. WJD, YC, and SGY performed the trilineage differentiation assay. YC performed endothelial differentiation assay. WJD, YC, ZXY, BQS, and XL performed RT$P C R$ and immunochemical staining. WJD performed the paracrine assay. WJD and ZXY performed the ELISA assay. ZCH, ZBH, and ZJL

participated in data analysis and manuscript writing. All authors revised their corresponding content and approved the final manuscript.

\section{Competing interests}

The authors declare that they have no competing interests.

\section{Consent for publication}

Not applicable.

\section{Ethics approval and consent to participate}

The study of human bone marrow-derived, adipose tissue-derived, umbilical cord-derived, and placental chorionic villi-derived MSCs has been approved by The Ethical Committee and The Institutional Review Board of Chinese Academy of Medical Science \& Peking Union Medical College. All donors signed an informed consent.

\section{Author details}

${ }^{1}$ The State Key Laboratory of Experimental Hematology, Institute of Hematology and Hospital of Blood Disease, Chinese Academy of Medical Science \& Peking Union Medical College, No. 288, Nanjing Road, Heping District, Tianjin 300020, China. ${ }^{2}$ Beijing Institute of Health and Stem Cells, No. 1, Kangding Road, BDA, Beijing 100176, China.

Received: 6 July 2016 Revised: 6 September 2016 Accepted: 4 October 2016 Published online: 10 November 2016

\section{References}

1. Uccelli A, Moretta L, Pistoia V. Mesenchymal stem cells in health and disease. Nat Rev Immunol. 2008:8(9):726-36.

2. Karantalis V, Suncion-Loescher VY, Bagno L, Golpanian S, Wolf A, Sanina C, et al. Synergistic effects of combined cell therapy for chronic ischemic cardiomyopathy. J Am Coll Cardiol. 2015;66(18):1990-9.

3. Huang PP, Yang XF, Li SZ, Wen JC, Zhang Y, Han ZC. Randomised comparison of G-CSF-mobilized peripheral blood mononuclear cells versus bone marrow-mononuclear cells for the treatment of patients with lower limb arteriosclerosis obliterans. Thromb Haemost. 2007:98(6):1335-42.

4. Ballotta V, Smits Al, Driessen-Mol A, Bouten CV, Baaijens FP. Synergistic protein secretion by mesenchymal stromal cells seeded in 3D scaffolds and circulating leukocytes in physiological flow. Biomaterials. 2014;35(33):9100-13.

5. Friedenstein AJ, Gorskaja JF, Kulagina NN. Fibroblast precursors in normal and irradiated mouse hematopoietic organs. Exp Hematol. 1976;4(5):267-74.

6. Bieback K, Brinkmann I. Mesenchymal stromal cells from human perinatal tissues: from biology to cell therapy. World J Stem Cells. 2010;2(4):81-92.

7. Heo JS, Choi Y, Kim HS, Kim HO. Comparison of molecular profiles of human mesenchymal stem cells derived from bone marrow, umbilical cord blood, placenta and adipose tissue. Int J Mol Med. 2016;37(1):115-25.

8. Wagner W, Wein F, Seckinger A, Frankhauser M, Wirkner U, Krause U, et al. Comparative characteristics of mesenchymal stem cells from human bone marrow, adipose tissue, and umbilical cord blood. Exp Hematol. 2005;33(11):1402-16. 
9. Baksh D, Yao R, Tuan RS. Comparison of proliferative and multilineage differentiation potential of human mesenchymal stem cells derived from umbilical cord and bone marrow. Stem Cells. 2007;25(6):1384-92.

10. Peltzer J, Montespan F, Thepenier C, Boutin L, Uzan G, Rouas-Freiss N, et al. Heterogeneous functions of perinatal mesenchymal stromal cells require a preselection before their banking for clinical use. Stem Cells Dev. 2015;24(3):329-44.

11. Liu M, Yang SG, Shi L, Du WT, Liu PX, Xu J, et al. Mesenchymal stem cells from bone marrow show a stronger stimulating effect on megakaryocyte progenitor expansion than those from non-hematopoietic tissues. Platelets. 2010;21(3):199-210.

12. Iwase T, Nagaya N, Fujii T, Itoh T, Murakami S, Matsumoto T, et al. Comparison of angiogenic potency between mesenchymal stem cells and mononuclear cells in a rat model of hindlimb ischemia. Cardiovasc Res. 2005;66(3):543-51.

13. van Velthoven CT, Kavelaars A, Heijnen CJ. Mesenchymal stem cells as a treatment for neonatal ischemic brain damage. Pediatr Res. 2012;71(4 Pt 2):474-81.

14. Li J, Zhao Q, Xing W, Feng J, Wu H, Li H, et al. Interleukin-27 enhances the production of tumour necrosis factor-alpha and interferon-gamma by bone marrow $\mathrm{T}$ lymphocytes in aplastic anaemia. $\mathrm{Br} \mathrm{J}$ Haematol. 2011;153(6):764-72.

15. Lu LL, Liu YJ, Yang SG, Zhao QJ, Wang X, Gong W, et al. Isolation and characterization of human umbilical cord mesenchymal stem cells with hematopoiesis-supportive function and other potentials. Haematologica. 2006;91(8):1017-26.

16. Wang Y, Zhang Z, Chi Y, Zhang Q, Xu F, Yang Z, et al. Long-term cultured mesenchymal stem cells frequently develop genomic mutations but do not undergo malignant transformation. Cell Death Dis. 2013;4:e950.

17. Pankajakshan D, Kansal V, Agrawal DK. In vitro differentiation of bone marrow derived porcine mesenchymal stem cells to endothelial cells. J Tissue Eng Regen Med. 2013;7(11):911-20.

18. Kim SW, Zhang HZ, Kim CE, An HS, Kim JM, Kim MH. Amniotic mesenchymal stem cells have robust angiogenic properties and are effective in treating hindlimb ischaemia. Cardiovasc Res. 2012;93(3):525-34.

19. Wu Y, Chen L, Scott PG, Tredget EE. Mesenchymal stem cells enhance wound healing through differentiation and angiogenesis. Stem Cells. 2007;25(10):2648-59.

20. Aguilera V, Briceno L, Contreras H, Lamperti L, Sepulveda E, Diaz-Perez F, et al. Endothelium trans differentiated from Wharton's jelly mesenchymal cells promote tissue regeneration: potential role of soluble pro-angiogenic factors. PLoS One. 2014:9(11):e111025.

21. Benavides OM, Petsche JJ, Moise Jr KJ, Johnson A, Jacot JG. Evaluation of endothelial cells differentiated from amniotic fluid-derived stem cells. Tissue Eng Part A. 2012;18(11-12):1123-31.

22. Chen MY, Lie PC, Li ZL, Wei X. Endothelial differentiation of Wharton's jellyderived mesenchymal stem cells in comparison with bone marrow-derived mesenchymal stem cells. Exp Hematol. 2009;37(5):629-40.

23. Ponce ML. Tube formation: an in vitro matrigel angiogenesis assay. Methods Mol Biol. 2009;467:183-8

24. Annabi B, Lee YT, Turcotte S, Naud E, Desrosiers RR, Champagne M, et al. Hypoxia promotes murine bone-marrow-derived stromal cell migration and tube formation. Stem Cells. 2003;21(3):337-47.

25. Hsiao ST, Asgari A, Lokmic Z, Sinclair R, Dusting GJ, Lim SY, et al. Comparative analysis of paracrine factor expression in human adult mesenchymal stem cells derived from bone marrow, adipose, and dermal tissue. Stem Cells Dev. 2012;21(12):2189-203.

26. Bronckaers A, Hilkens P, Martens W, Gervois P, Ratajczak J, Struys T, et al. Mesenchymal stem/stromal cells as a pharmacological and therapeutic approach to accelerate angiogenesis. Pharmacol Ther. 2014;143(2):181-96.

27. Nagasaki T, Hara M, Nakanishi H, Takahashi H, Sato M, Takeyama H. Interleukin-6 released by colon cancer-associated fibroblasts is critical for tumour angiogenesis: anti-interleukin-6 receptor antibody suppressed angiogenesis and inhibited tumour-stroma interaction. Brit J Cancer. 2014;110(2):469-78.

28. Simonini A, Moscucci M, Muller DW, Bates ER, Pagani FD, Burdick MD, et al. $\mathrm{LL}-8$ is an angiogenic factor in human coronary atherectomy tissue. Circulation. 2000;101(13):1519-26.

29. Salmeron K, Aihara T, Redondo-Castro E, Pinteaux E, Bix G. IL-1alpha induces angiogenesis in brain endothelial cells in vitro: implications for brain angiogenesis after acute injury. J Neurochem. 2015. doi:10.1111/jnc.13422.
30. Sola-Villa D, Camacho M, Sola R, Soler M, Diaz JM, Vila L. IL-1beta induces VEGF, independently of PGE2 induction, mainly through the PI3-K/mTOR pathway in renal mesangial cells. Kidney Int. 2006;70(11):1935-41.

31. Gately S. The contributions of cyclooxygenase-2 to tumor angiogenesis. Cancer Metastasis Rev. 2000;19(1-2):19-27.

32. Zhang Y, Daaka Y. PGE2 promotes angiogenesis through EP4 and PKA Cgamma pathway. Blood. 2011;118(19):5355-64.

33. Kwon HM, Hur SM, Park KY, Kim CK, Kim YM, Kim HS, et al. Multiple paracrine factors secreted by mesenchymal stem cells contribute to angiogenesis. Vascul Pharmacol. 2014;63(1):19-28.

34. Amable PR, Teixeira MV, Carias RB, Granjeiro JM, Borojevic R. Protein synthesis and secretion in human mesenchymal cells derived from bone marrow, adipose tissue and Wharton's jelly. Stem Cell Res Ther. 2014;5(2):53.

35. Ikhapoh IA, Pelham CJ, Agrawal DK. Atherogenic cytokines Regulate VEGFA-induced differentiation of bone marrow-derived mesenchymal stem cells into endothelial cells. Stem Cells Int. 2015;2015:498328.

36. Choi M, Lee HS, Naidansaren $\mathrm{P}$, Kim HK, Eunju O, Cha JH, et al. Proangiogenic features of Wharton's jelly-derived mesenchymal stromal/stem cells and their ability to form functional vessels. Int J Biochem Cell Biol. 2013;45(3):560-70.

37. Janeczek Portalska K, Leferink A, Groen N, Fernandes H, Moroni L, van Blitterswijk $C$, et al. Endothelial differentiation of mesenchymal stromal cells. PLoS One. 2012;7(10):e46842.

38. Wang $H$, Riha GM, Yan S, Li M, Chai H, Yang H, et al. Shear stress induces endothelial differentiation from a murine embryonic mesenchymal progenitor cell line. Arterioscler Thromb Vasc Biol. 2005;25(9):1817-23.

39. Zhang $X$, Nan $Y$, Wang $H$, Chen J, Wang N, Xie J, et al. Model microgravity enhances endothelium differentiation of mesenchymal stem cells. Naturwissenschaften. 2013;100(2):125-33.

40. Moon MH, Kim SY, Kim YJ, Kim SJ, Lee JB, Bae YC, et al. Human adipose tissue-derived mesenchymal stem cells improve postnatal neovascularization in a mouse model of hindlimb ischemia. Cell Physiol Biochem. 2006;17(5-6):279-90.

41. Rehman J, Traktuev D, Li J, Merfeld-Clauss S, Temm-Grove CJ, Bovenkerk JE, et al. Secretion of angiogenic and antiapoptotic factors by human adipose stromal cells. Circulation. 2004;109(10):1292-8.

42. Kandel ER, Pittenger $C$. The past, the future and the biology of memory storage. Philos Trans R Soc Lond B Biol Sci. 1999;354(1392):2027-52.

43. Nor JE, Christensen J, Mooney DJ, Polverini PJ. Vascular endothelial growth factor (VEGF)-mediated angiogenesis is associated with enhanced endothelial cell survival and induction of $\mathrm{BCl}-2$ expression. Am J Pathol. 1999;154(2):375-84.

44. Bussolino F, Di Renzo MF, Ziche M, Bocchietto E, Olivero M, Naldini L, et al. Hepatocyte growth factor is a potent angiogenic factor which stimulates endothelial cell motility and growth. J Cell Biol. 1992;119(3):629-41.

45. Rice GE, Bevilacqua MP. An inducible endothelial cell surface glycoprotein mediates melanoma adhesion. Science. 1989;246(4935):1303-6.

46. Castrechini NM, Murthi P, Gude NM, Erwich JJ, Gronthos S, Zannettino A, et al. Mesenchymal stem cells in human placental chorionic villi reside in a vascular Niche. Placenta. 2010;31(3):203-12.

47. Levesque JP, Takamatsu Y, Nilsson SK, Haylock DN, Simmons PJ. Vascular cell adhesion molecule-1 (CD106) is cleaved by neutrophil proteases in the bone marrow following hematopoietic progenitor cell mobilization by granulocyte colony-stimulating factor. Blood. 2001;98(5):1289-97.

48. Winkler IG, Sims NA, Pettit AR, Barbier V, Nowlan B, Helwani F, et al. Bone marrow macrophages maintain hematopoietic stem cell (HSC) niches and their depletion mobilizes HSCs. Blood. 2010;116(23):4815-28.

49. Wang CM, Guo Z, Xie YJ, Hao YY, Sun JM, Gu J, et al. Co-treating mesenchymal stem cells with IL 1 beta and TNF-alpha increases VCAM-1 expression and improves post-ischemic myocardial function. Mol Med Rep. 2014;10(2):792-8.

50. Du W, Li X, Chi Y, Ma F, Li Z, Yang S, et al. VCAM-1(+) placenta chorionic villi-derived mesenchymal stem cells display potent pro-angiogenic activity. Stem Cell Res Ther. 2016;7(1):49.

51. Konig J, Weiss G, Rossi D, Wankhammer K, Reinisch A, Kinzer M, et al. Placental mesenchymal stromal cells derived from blood vessels or avascular tissues: what is the better choice to support endothelial cell function? Stem Cells Dev. 2015;24(1):115-31.

52. Jeon YJ, Kim J, Cho JH, Chung HM, Chae Jl. Comparative analysis of human mesenchymal stem cells derived from bone marrow, placenta, and adipose tissue as sources of cell therapy. J Cell Biochem. 2016:117(5):1112-25. 